\title{
PROGRAMAÇÃO WEB PARA DESENVOLVIMENTO DE SOFTWARE EDUCACIONAL COMO FERRAMENTA DE ENSINO NA ENGENHARIA CIVIL
}

Rodrigo Galardo Neves

Graduando em Engenharia Civil pelo Centro Federal de Educação Tecnológica Celso Suckow da Fonseca (CEFET/RJ), Rio de Janeiro, RJ, Brasil rodrigo.neves@aluno.cefet-rj.br

Ricardo Rodrigues de Araujo Professor pelo Centro Federal de Educação Tecnológica Celso Suckow da Fonseca (CEFET/RJ), Rio de Janeiro, RJ, Brasil ricardo.araujo@cefet-rj.br

\section{RESUMO}

Este projeto visa o desenvolvimento de software com finalidades acadêmicas para a engenharia civil, de modo a possibilitar um fácil acesso a qualquer estudante, sem necessidade de instalação por parte do usuário final e uma interface intuitiva. Para tal, o autor participa de cursos online e gratuitos sobre programação web, abordando o assunto dentro da engenharia civil (perspectiva de risco de incidentes em taludes de rodovias). Realizam-se cálculos a partir de parâmetros que relativos a características referentes ao talude avaliado e considerando probabilidade e danos. Assim, obtém-se uma página web com entrada de dados pelo usuário, que pode, com esta, estudar e se familiarizar com o assunto e as possibilidades aplicadas no estudo, tanto na análise abordada quanto nos resultados gerados pelo programa.

Palavras-chave: Contenção de taludes. Desenvolvimento de software. Segurança rodoviária. Terraplenagem.

\section{WEB PROGRAMMING FOR DEVELOPMENT OF EDUCATIONAL SOFTWARE AS A LEARNING TOOL IN CIVIL ENGINEERING}

\begin{abstract}
This project aims to develop a software with academic purposes for civil engineering, to allow easy access to any student, without the need of installation by the user and an intuitive interface. To achieve this, the author takes free online courses in web programming, relating to the subject within civil engineering (perspective of risk of incidents on highway embankments). Calculations are made based on parameters related to characteristics of the evaluated slope and considering probability and damage. As a result, it is obtained a web page with data entry for the user, who can study and become familiar with the subject and the possibilities applied in the study, both in the analysis and in the results generated by the program.
\end{abstract}

Keywords: Slope control. Software development. Road safety. Earthwork. 


\section{INTRODUÇÃO}

A ação antropogênica de implantação de terraplenos rodoviários não pode ser considerada um fim em si, pois é dinâmica no tempo, visto que as rochas e os solos estão num processo de transformação ininterrupto, passando por desgaste natural e fatores constantes de intemperismo. Para evitar a instabilidade dos terraplenos é necessário realizar a monitoração anual, para diagnóstico de problemas.

O monitoramento deve avaliar o Risco de Incidentes, sendo Risco = Probabilidade de ocorrência $\times$ danos ou consequência; e Incidente, a ocorrência de emergência com o usuário da via, podendo ser desastre natural ou evento não planejado que afete ou impeça o fluxo normal de tráfego.

Com a perspectiva de garantir condições seguras para os usuários da rodovia, em relação a instabilidade de terraplenos, foi desenvolvida a metodologia de Perspectiva de Risco de Incidentes - PRI. A PRI é de fácil aplicação, podendo ser utilizada com os valores "default" propostos ou valores diversos, conforme a perspectiva do especialista responsável pela avaliação do conjunto de terraplenos.

Como input, a PRI utiliza oito parâmetros, sendo inseridos valores geométricos e características dos terraplenos; como output são estabelecidos níveis de risco para cada terrapleno e a consequente proposta de intervenção no período (DER; IPT, 1991).

\section{METODOLOGIA}

O passo inicial para o desenvolvimento do projeto é o estudo de programação web. Para tal, após conhecimento prévio em lógica de programação, é necessária a abordagem de diferentes linguagens de programação, sendo estas HTML, CSS e PHP. Deste modo, foram indicados pelo orientador alguns cursos gratuitos online desenvolvidos pelo site Curso em Vídeo.

O primeiro curso aborda HTML (HyperText Markup Language) de forma geral enquanto se desenvolve um site simples. Códigos HTML representam o conteúdo de páginas web, como textos, imagens, tabelas, divisões e referências internas a serem utilizadas por desenvolvedores e outras linguagens de programação. O curso também explica a lógica de CSS (Cascading Style Sheets) e apresenta algumas possibilidades básicas de formatação de textos, tamanhos, cores e outros elementos de design, além de usar uma funcionalidade básica de JavaScript, com o qual é possível alterar elementos da página separadamente. 
O segundo apresenta a linguagem PHP (Hypertext Preprocessor), com a qual uma página pode realizar cálculos e exibir conteúdos dependentes de parâmetros, dentre outras possibilidades. São apresentadas noções de variáveis, operadores, integração com HTML, estruturas condicionais, de repetição, de strings (cadeias de caracteres), vetores e matrizes. A separação por sequências e métodos é essencial para a realização do programa proposto.

No último curso, estuda-se a abordagem de POO (Programação Orientada a Objetos) em PHP. O curso é separado em duas partes alternadas, sendo uma de conceitos gerais de POO em algoritmo e outra a aplicação prática destes conceitos na linguagem PHP. Iniciando-se com a introdução ao assunto, o curso abordou os temas de definições de classes e objetos, visibilidade de atributos e métodos, os conceitos de encapsulamento, herança e polimorfismo. Tais assuntos ajudam na otimização dos códigos e processos utilizados.

O objetivo do software, inicialmente, é de calcular a PRI (perspectiva de risco de incidentes) de taludes em faixa de domínio de rodovias. Consiste em oito características, sendo estas altura, distância do acostamento, drenagem, extensão, inclinação, obras de contenção, rochas e vegetação; devendo o usuário marcar um dos parâmetros entre I, II, III ou IV (descritos no software) e admitidos pesos para cada um desses. O cálculo é efetuado pelo programa que apresenta na tela o valor final encontrado $\left(P R I_{b}\right)$, a perspectiva (baixa, média, alta ou muito alta) e uma breve descrição das medidas e prazos a serem adotados. A fórmula consiste na soma dos produtos entre o peso do parâmetro selecionado e a importância de cada uma das oito categorias.

Posteriormente, com o decorrer dos estudos da academia no assunto, define-se que cada característica deve possuir uma importância percentual. Além disso, os parâmetros de características diferentes podem ter pesos diferentes entre si. No programa, então, as importâncias podem ser alteradas pelo usuário ou mantido o padrão; pode-se manter padrão ou alterar uma série geral dos pesos; e possibilitar ao usuário mudar pesos de características individuais, caso desejado. Os intervalos de valores para $\mathrm{PRI}_{\mathrm{b}}$ que definem a perspectiva (de baixa a muito alta) também são padronizados, mas alteráveis no programa.

Com isso, o cálculo do $\mathrm{PRI}_{\mathrm{b}}$ é feito da seguinte maneira:

$$
P R I_{b}=\frac{\sum_{i=1}^{8} P_{i} \cdot I_{i}}{\sum_{i=1}^{8} I_{i}}
$$

Sendo $i$ um índice para as oito características, $P$ o peso escolhido e $I$ a importância definida para cada característica.

O software consiste em quatro arquivos PHP ("Calculador.php", "Caracteristica.php", “index.php" e "resultados.php"), um arquivo CSS ("estilo.css") e uma pasta contendo três imagens 
(“1.jpeg", "2.jpeg" e "3.jpeg"). “Calculador.php" e “Caracteristica.php" são arquivos de objetos, onde são realizadas as funções do programa; "index.php" e "resultados.php", arquivos acessados diretamente pelo usuário, são a interface de entrada dos dados e de visualização dos resultados, respectivamente; "estilo.css" define o estilo das interfaces (figuras 1 e 2); e as imagens (figuras 3, 4 e 5) são utilizadas para ilustrar o tema.

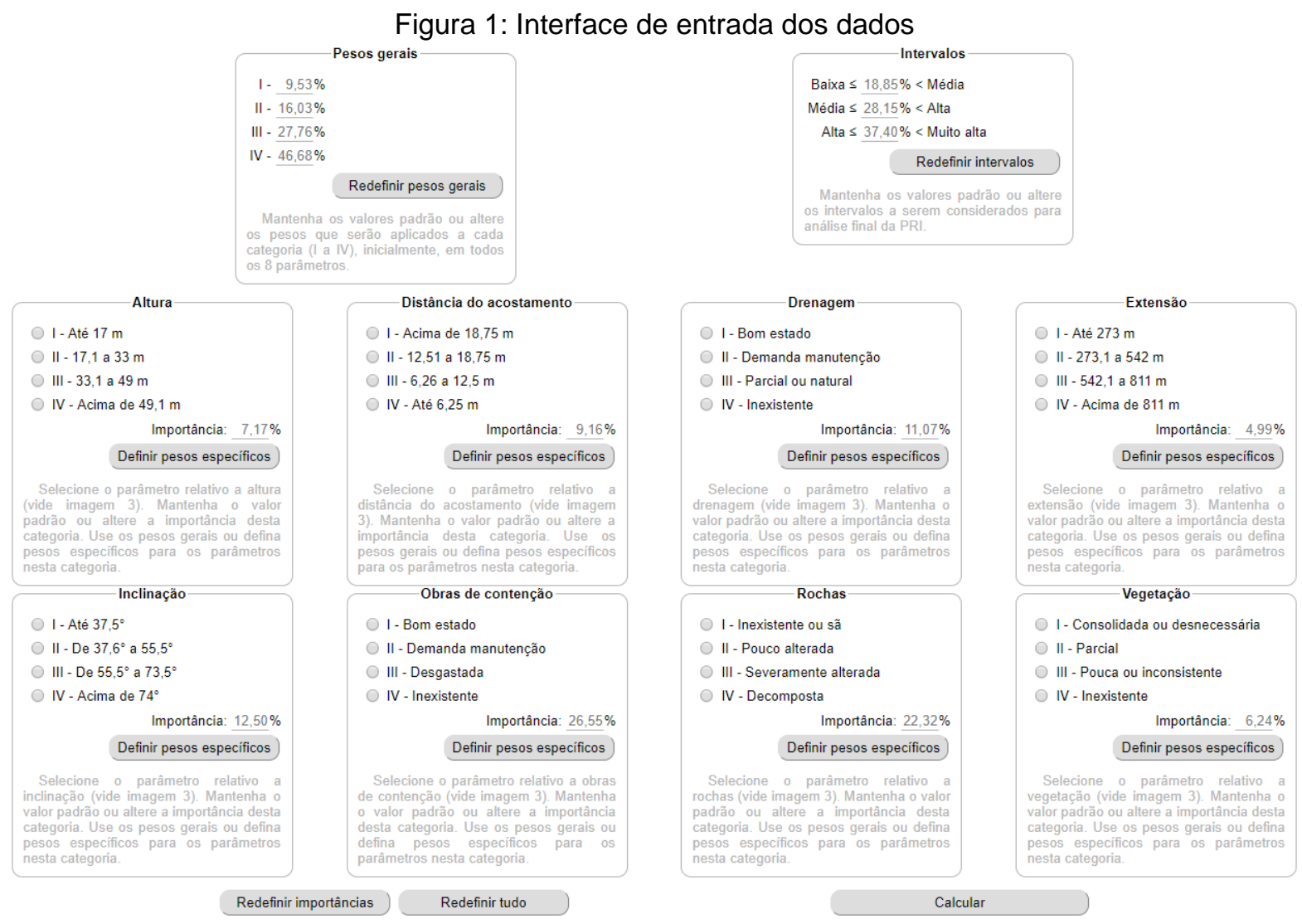

Fonte: O autor (2019) 
Figura 2: Interface de apresentação dos resultados

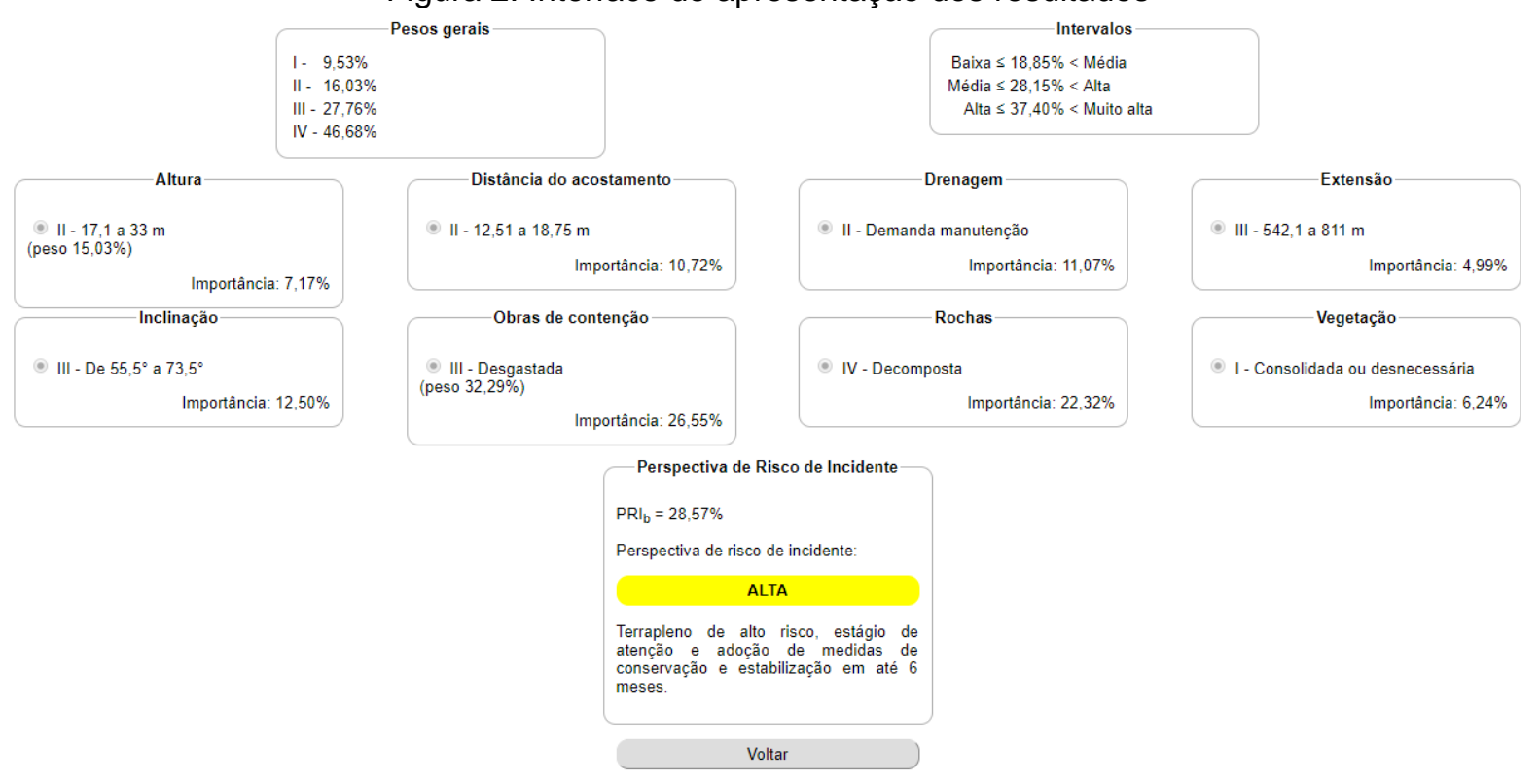

Fonte: O autor (2019)

Figura 3: Exemplo de talude com dispositivos de drenagem

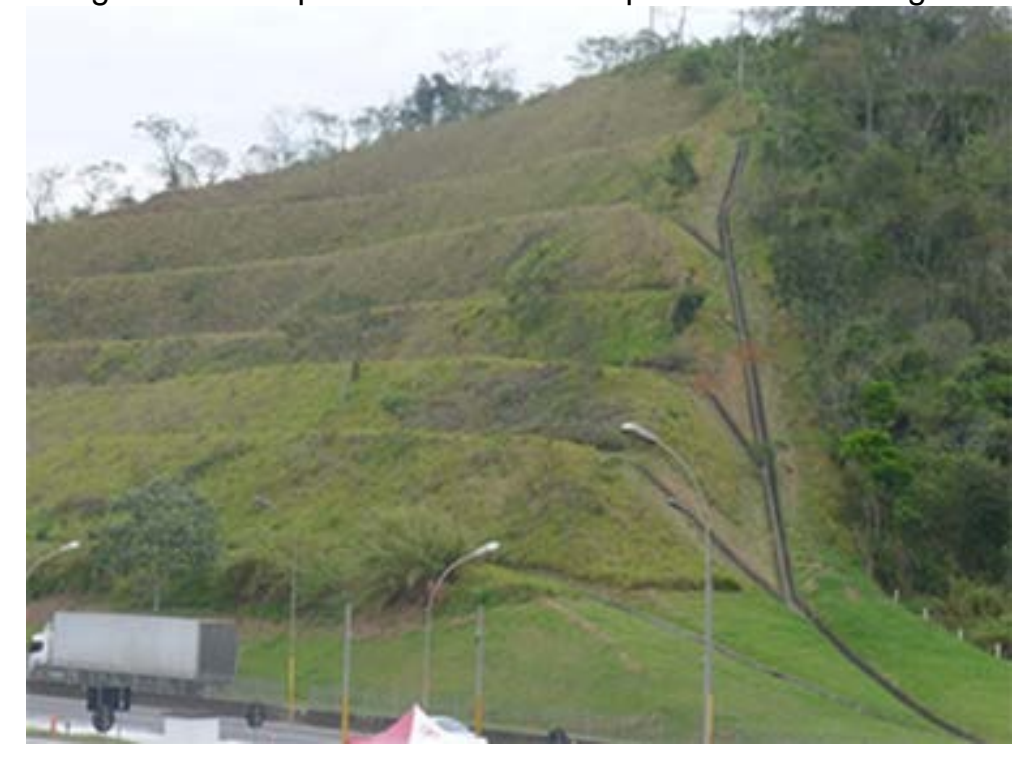

Fonte: SILVA (2019) 
Figura 4: Exemplo de talude de pequenas dimensões de extensão e altura

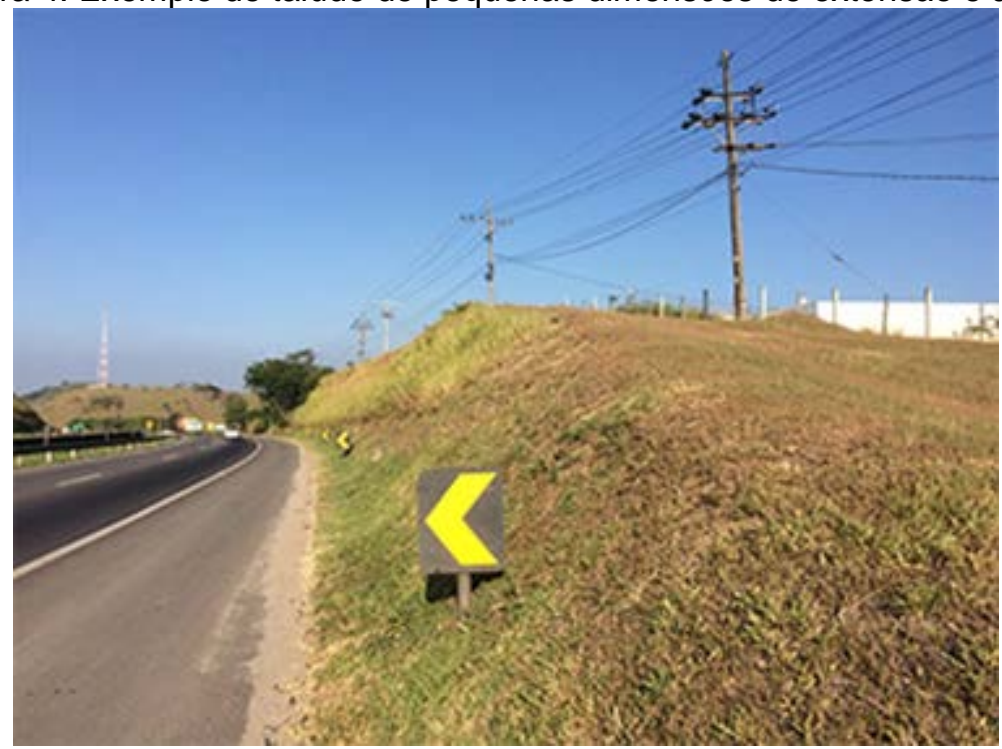

Fonte: SILVA (2019)

Figura 5: Desenho esquemático representando as oito características abordadas

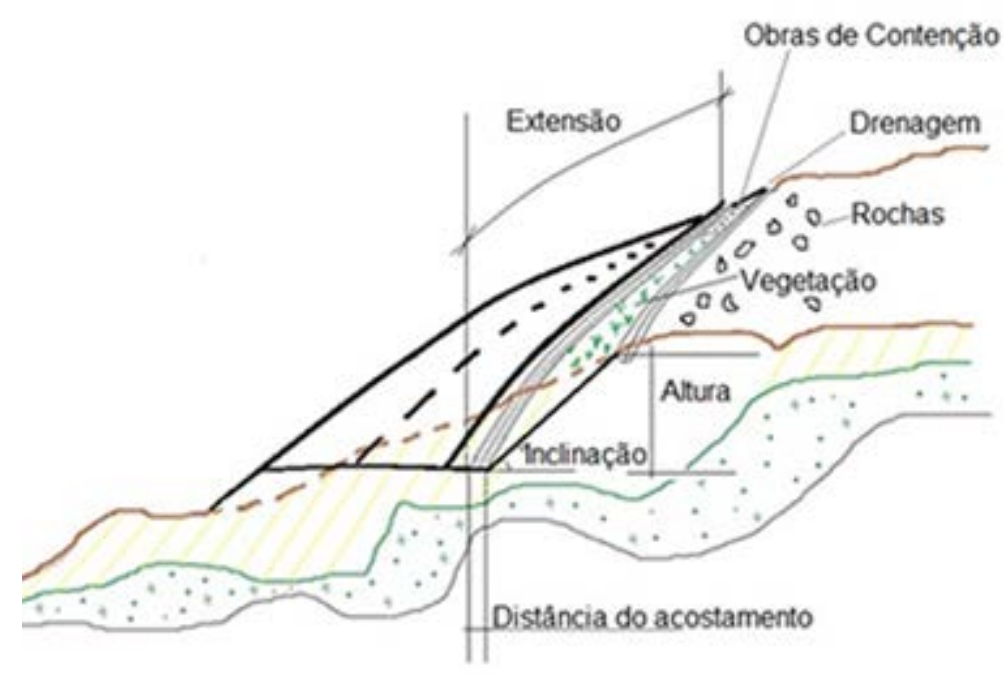

Fonte: SILVA (2019)

\section{RESULTADOS}

Os resultados calculados, como explicado anteriormente, são o valor $\mathrm{PRI}_{\mathrm{b}}$, a perspectiva correspondente e uma explicação sobre medidas e prazos a serem tomados, como apresentado na figura 6. No entanto, deve-se sempre considerar as possibilidades de erros. A inserção de números só aceita valores acima de zero e menores ou iguais a 100\%, assim evitando divisão por zero e cálculos exagerados. Caso a soma das importâncias inseridas das categorias não seja de 100\%, é feita uma correção proporcional. Caso alguma característica não tenha sido marcada e/ou os 
valores dos pesos ou dos intervalos de análise não estejam em ordem crescente, o cálculo não é efetuado e o programa indica na tela apenas o(s) erro(s) ocorrido(s), como os vistos na figura 7.

Figura 6: Possível resultado calculado e apresentado pelo programa

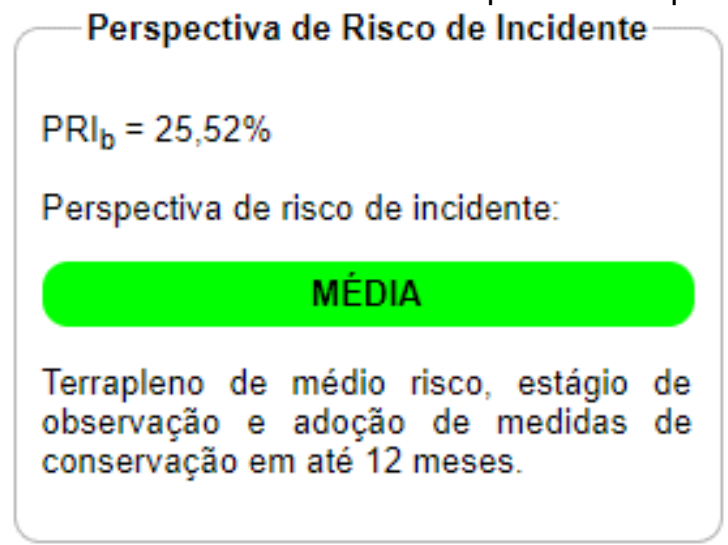

Fonte: O autor (2019)

Figura 7: Possíveis erros de inserção de valores

Pesos gerais inválidos. Insira valores em
ordem crescente.
Intervalos inválidos. Insira valores em
ordem crescente.
Característica(s) não definida(s).
Selecione todas as características
pedidas.

Fonte: O autor (2019)

\section{CONCLUSÃO}

O programa é acessível por qualquer estudante com acesso à internet por um navegador web, em interface intuitiva, com segurança e sem necessidade de instalação. Com seu uso, é possível entender quais são as características analisadas em taludes rodoviários e quanto estas influenciam na segurança de uma rodovia, as noções de urgência de intervenção, além de auxiliar nas pesquisas sobre o assunto ao facilitar alterações de parametrização.

Foram realizados testes para diferentes possibilidades e singularidades com exibição de etapas de cálculo, que, junto com contas paralelas em outras calculadoras, confirmam o sucesso dos cálculos pelo software. A tentativa não sucedida de gerar erros no sistema, a impossibilidade 
de se inserir valores desfavoráveis ao estudo e a previsão em código de erros comuns mostram a eficácia do algoritmo.

Além disso, o design simples e minimalista facilita e deixa seu uso intuitivo. O programa pesa apenas $653 \mathrm{kB}$, sendo $23 \mathrm{kB}$ de códigos e o restante das imagens. Sendo disponibilizado na internet, o programa torna-se democrático e acessível para qualquer estudante.

\section{REFERÊNCIAS}

DEPARTAMENTO DE ESTRADAS DE RODAGEM DO ESTADO DE SÃO PAULO; INSTITUTO DE PESQUISAS TECNOLÓGICAS. Manual de Geotecnia: Taludes de Rodovias - Orientação para Diagnóstico e Soluções de seus Problemas. São Paulo, 1991.

PINTO, C. D. S. Curso básico de mecânica dos solos em 16 aulas. 3a ed. São Paulo: Oficina de Textos, 2006.

SILVA, C. A. S. Aplicação da Sistemática de Perspectiva de Risco de Incidentes em Terraplenos, na Monitoração Anual de Rodovias. Rio de Janeiro, 2019.

SILVA, M. A. Aplicação de Lógica Nebulosa para Previsão do Risco de Escorregamentos de Taludes em Solo Residual. Rio de Janeiro: UERJ, 2008 